\title{
PREDICTION OF SITE RESPONSE SPECTRUM UNDER EARTHQUAKE VIBRATION USING AN OPTIMIZED DEVELOPED ARTIFICIAL NEURAL NETWORK MODEL
}

\author{
Reza Esmaeilabadi', Abbas Abbaszadeh Shahri² \\ 1 Department of Civil Engineering, College of Civil Engineering, Roudehen Branch, Islamic Azad University, \\ Roudehen, Tehran, Iran, e-mail: esmaeilabadi@riau.ac.ir \\ 2 Department of Civil and Architectural Engineering, KTH Royal Institute of Technology, Stockholm, Sweden, \\ e-mail: a_abbaszadeh@iauh.ac.ir
}

Received: 2016.04.05

Accepted: 2016.05.10

Published: 2016.06.01

\begin{abstract}
Site response spectrum is one of the key factors to determine the maximum acceleration and displacement, as well as structure behavior analysis during earthquake vibrations. The main objective of this paper is to develop an optimized model based on artificial neural network (ANN) using five different training algorithms to predict nonlinear site response spectrum subjected to Silakhor earthquake vibrations is. The model output was tested for a specified area in west of Iran. The performance and quality of optimized model under all training algorithms have been examined by various statistical, analytical and graph analyses criteria as well as a comparison with numerical methods. The observed adaptabilities in results indicate a feasible and satisfactory engineering alternative method for predicting the analysis of nonlinear site response.
\end{abstract}

Keywords: nonlinear, site response spectrum, optimized ANN model, Iran, analyses criteria.

\section{INTRODUCTION}

Site (ground) response spectrum is a nonlinear plot of the peak value of a response quantity (e.g. acceleration) in the earth surface as a function of the vibration period of the system and depends on the damping ratio and the selected ground motion. Significant seismic damage may occur if the building response is in resonance with components of the ground motion, which may be identified from the response spectrum. The impedance ratio between surface strata and the underlying bedrock as well as surface topography can affect the site response during a severe earthquake. However, nonlinear site response describes a situation when a site responds differently depending upon the strength of shaking [1-7]. The nonlinear site response during earthquake vibrations can be influenced by geological deposits and local soil conditions [7-12]. Large earthquakes with particularly strong vibrations and a characterized compliant medium are necessary conditions for a nonlinear site response [1]. However, obtaining site response spectra due to soil nonlinearity, the unavoidable uncertainties as well as adopted simplifications during the design process can be an imprecise scientific field [13]. Moreover, the available conventional computer programs have an inherent limitation due to their sequential and algorithmic approach. To overcome this problem, relatively accurate predictions using advanced soft computing and, in particular, the artificial neural network (ANNs) approach in geo-engineering applications and in particular to site response characterization can be tolerated rather than solving a problem conventionally [14-24]. The efficient handling of highly nonlinear relationships in data, even in unknown 
exact nature of such relationship is one of the major advantages of ANNs. Therefore, the ANNs can easily form models for complex problems as well as successfully application in learning related classification, generalization, characterization and optimization functions.

In this paper a Matlab computer code based on several different training algorithms as well as various activation transfer functions have been developed to find an optimized ANN model to predict the site response spectra for a specified high risk seismic zone under Silakhor earthquake vibrations (Ms6.1, 2006, Iran). Among the tested ANN training algorithms, the conjugate gradient descent showed better performance based on the employed criteria. In the introduced model, the importance of the adequate soil behavior using the in-situ and laboratory tests as well as geophysical servying have been considered to simulate earthquake site response spectra. The conducted comparison between the ANN results with a previous study [25] and time domain nonlinear method highlighted an attractive economical engineering based alternative method that can cover and solve some limitations of the conventional methods.

\section{TARGET SITE AND USED DATASETS}

The Hamedan province (Fig. 1B) is situated in Zagros mountain fold-and-thrust belt with NW-SE strike (Fig. 1A) as the most seismically active belts in west of Iran with frequently recorded medium to large magnitude earthquakes [26, 27]. The target area in this paper (Fig. 1B and C) is the Korzan earth dam site with $43 \mathrm{~m}$ height from the river bed and $1,428 \mathrm{~m}$ crest line length with $2 \mathrm{Km}$ distance from Korzan village and $10 \mathrm{Km}$ from Tuyserkan city (Fig. 1B). The site of the dam is located at $34^{\circ} 34^{\prime} 20^{\prime \prime}$ to $34^{\circ} 35^{\prime}$ north latitude and $48^{\circ} 20^{\prime}$ to $48^{\circ} 23^{\prime} 10^{\prime \prime}$ east longitude and has been subjected to earthquake geotechnical analysis [25].

Considering the importance of accuracy, completeness, consistency and quality of data on ANNs output [28], data collection plays an important and significant role. Therefore, in this paper, the previous databases [25] have been updated using geo-mechanical data and geophysical surveying form field tests and other relevant sources and were categorized into four main subcategories by the authors, as drilled borelog data (e.g. soil layers, soil types, layer thickness, depth to bedrock level), field and laboratory test data (e.g. standard penetration test (SPT), sieve analysis, unit weight, shear wave velocity $\left(\mathrm{V}_{\mathrm{S}}\right)$, shear modulus, plasticity, permeability, degree of saturation, cohesion of the soil, ground water table, pore pressure), computed data (e.g. total and effective vertical stress $\left(\sigma_{v}, \sigma_{v}^{\prime}\right)$, damping, and stress reduction factor $\left(\mathrm{r}_{\mathrm{d}}\right)$ ) and recorded data of Silakhor earthquake (Ms6.1, 2006, Iran) at Tuyserkan station as input motion.

The epicenter of event and recorded peak ground acceleration (PGA) respected to studied area is presented in Figure 1C. The depths of drilled borehole vary between 30 to $80 \mathrm{~m}$ and ground water table found between 1.3 to $20 \mathrm{~m}$ respectively.

The complexity of geological soil deposit structures causes a highly nonlinear behavior in site response analysis which is contributed to quantitative physical parameters, such as $\mathrm{V}_{\mathrm{S}}$ and damping factors $[29,30]$. Therefore, it is necessary to know the soil related properties and the variability in $\mathrm{V}_{\mathrm{S}}$ with change in soil properties.

\section{ANN AND BACK PROPAGATION LEARNING ALGORITHM}

The ANNs are novel developed computational models of the information processing system based on the biological nervous system. They are

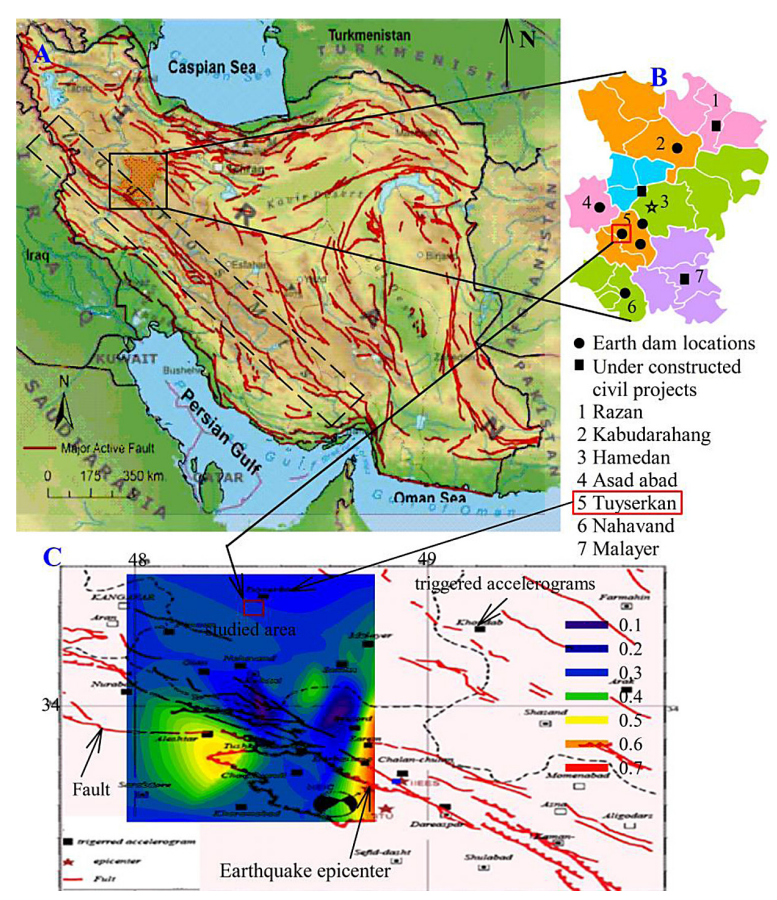

Fig. 1. (A) Location of Hamedan province respect to Zagros main recent fault in Iran, (B) Situation of target area and other available earth dams, (C) PGA contour line and epicenter of Silakhor earthquake (Ms6.1, 2006, Iran) respected to the studied area 
composed of a large number of highly interconnected processing elements (neurons) to solve specific problems [31]. The learning system of ANNs is one of the major differences compared to traditional statistical or rule-based systems [32]. In ANNs, the neurons are interconnected via a set of weights and suitable activation functions which play a major role in processing inputs and outputs. The way of interconnection among the processing elements determines the network architecture. The input layer projects the data to the intermediate (hidden) layers while the final hidden layer projects the information to the output neurons (Fig. 2). The final weights and thresholds of activation for decreasing the error between the observed and computed outputs subject to a sufficient level defined by the user are set in the training phase of the ANN algorithm.

As presented in Figure 2, the $X_{i}$ as the signal from the $i^{\text {th }}$ input is connected to another neuron $j$ with associated weight $\mathrm{w}_{\mathrm{ij}}$ between the $\mathrm{X}_{\mathrm{i}}$ and $\mathrm{X}_{\mathrm{j}}$. Eq. 1 is a result of multiplication and summation of the output of each neuron $\mathrm{i}$ by $\mathrm{w}_{\mathrm{i}, \mathrm{j}}$ by an associated bias $\left(\theta_{i}\right)$ to each connection link between input layer $\mathrm{i}$ and hidden layer $\mathrm{j}$.

$$
\text { Sum }=\sum_{i=1}^{n}\left(x_{i} w_{i}\right)+\theta_{i}
$$

The output of a neuron (y) as an activation function (f) of the weighted sum of $n+1$ inputs can be defined as Eq.2. These $\mathrm{n}+1$ correspond to the $\mathrm{n}$ incoming signals. The threshold is incorporated into Eq. 3 and the output of $\mathrm{k}^{\text {th }}$ neuron can be obtained by Eq. 4 .

$$
\begin{gathered}
y=f\left(\sum_{i=0}^{n} x_{i} w_{i}\right) \\
f(x)=\left\{\begin{array}{cc}
1 & \sum_{i=0}^{n} x_{i} w_{i}>0 \\
0 & \sum_{i=0}^{n} x_{i} w_{i} \leq 0
\end{array}\right. \\
\text { net }_{k}=\sum_{j}\left(O_{j} w_{j, k}\right)+\theta_{k}
\end{gathered}
$$

Using Eq.5, the mean square error (MSE) is used as a network error function to calculate the error at each iteration during the learning process.

$$
M S E=\frac{1}{2} \sum_{k}\left(t_{k}-O_{k}\right)^{2}
$$

where $t_{k}$ is the target output at layer $k$ and $\mathrm{O}_{k}$ is the final output at the output layer.

To decrease the error value, the derivative of MSE using the chain rule with respect to weight is computed and back-propagated to the layers to compute the new weight value [32] (Eq.6).

$$
\frac{\partial M S E}{\partial w_{i j}}=\frac{\partial M S E}{\partial a_{i}} \frac{\partial a_{i}}{\partial n e t_{j}} \frac{\partial n e t_{i}}{\partial w_{i j}}
$$

where $w_{i j}, a_{i}$ and net $t_{i}$ are weight from neuron $j$ to neuron $i$, activation value and weighted sum of the inputs of neuron I respectively.

This algorithm which uses the gradient descent method is known as the delta rule (Eq.7) [32]. Then the new weight value at $(\mathrm{t}+1)$ th iteration between output layer to hidden layer $\mathrm{j}$ can be calculated by Eq. 8 .

$$
\begin{aligned}
& \Delta w_{k j}(t)=-\eta \frac{\partial M S E}{\partial w_{k j}}+\beta \Delta w_{k j}(t-1) \\
& w_{k j}(t+1)=\Delta w_{k j}(t)+w_{k j}(t)
\end{aligned}
$$

where $\eta$ and $\beta$ are the learning and momentum parameters and $t$ is the number of iteration respectively.

\section{PROCEDURE TO INTRODUCE THE OPTIMIZED ANN MODEL STRUCTURE}

In the current paper, the depth, soil type, SPT, $\sigma \mathrm{v}, \sigma^{\prime} \mathrm{v}, \mathrm{rd}$ and VS due to their proved effect on site response spectrum were selected as model inputs. The procedure to find the optimized ANN structure to predict the nonlinear seismic site response spectrum was found through the trial and error method using a developed Matlab computer code. The optimized model is introduced by highest value of network correlations and minimum root mean square error (RMSE). By application of five training algorithms (quick propagation (QP), conjugate gradient descent (CGD), limited memory quasi Newton (LMQN), quasi Newton (QN) and Levenberg-Marquardt (L-M)) as well as various activation transfer functions, more than 560 topologies were tested, trained and developed and their performance was controlled using several statistical, analytical and graph analyses criteria.

The result of tested models showed that a four layers model with 7-7-5-5-3-3-1structure containing 23 neurons under CGD training algorithm and hyperbolic tangent activation function satisfy the minimum RMSE and highest network correlation (Figs 3, 4 and Tabs.1,2). In order to have a better view, for 23 neurons many structures such as 7-7-5-6-3-1, 7-6-7-5-3-1, 7-6-5-7-3-1, 7-46-6-7-1 were tested. For example, the structure of 7-7-5-6-3-1 was separately controlled for all training algorithms using hyperbolic tangent and 
Table 1. Characteristics of optimized ANN structure in this study based on tested algorithms

\begin{tabular}{|c|c|c|c|c|}
\hline \multirow{2}{*}{$\begin{array}{c}\text { ANN training } \\
\text { algorithm }\end{array}$} & \multirow{2}{*}{ Network error } & \multirow{2}{*}{ Number of iteration } & \multicolumn{2}{|c|}{ Activation function } \\
\cline { 3 - 5 } & & Hidden layer activation & Output activation \\
\hline QP & 0.00257 & 81 & logistic & logistic \\
\hline CGD & 0.0024 & 139 & hyperbolic tangent & hyperbolic tangent \\
\hline QN & 0.00296 & 79 & hyperbolic tangent & logistic \\
\hline LMQN & 0.0032 & 114 & logistic & hyperbolic tangent \\
\hline L-M & 0.0026 & 65 & hyperbolic tangent & hyperbolic tangent \\
\hline
\end{tabular}

Table 2. Network results of applied algorithms using the introduced optimized model in this paper

\begin{tabular}{|c|c|c|c|c|c|c|c|c|}
\hline \multirow{2}{*}{ Algorithm } & \multicolumn{2}{|c|}{ Train data } & \multicolumn{2}{c|}{ Test data } & \multicolumn{2}{c|}{ Validate data } & \multicolumn{2}{c|}{ All data } \\
\cline { 2 - 9 } & correlation & $\mathrm{R}^{2}$ & correlation & $\mathrm{R}^{2}$ & correlation & $\mathrm{R}^{2}$ & correlation & $\mathrm{R}^{2}$ \\
\hline QP & 0.861 & 0.812 & 0.863 & 0.833 & 0.868 & 0.841 & 0.871 & 0.834 \\
\hline CGD & 0.918 & 0.869 & 0.922 & 0.851 & 0.889 & 0.860 & 0.912 & 0.882 \\
\hline QN & 0.910 & 0.847 & 0.915 & 0.839 & 0.914 & 0.840 & 0.918 & 0.841 \\
\hline LMQN & 0.871 & 0.833 & 0.884 & 0.856 & 0.900 & 0.860 & 0.893 & 0.871 \\
\hline L-M & 0.912 & 0.841 & 0.921 & 0.843 & 0.920 & 0.842 & 0.919 & 0.845 \\
\hline
\end{tabular}

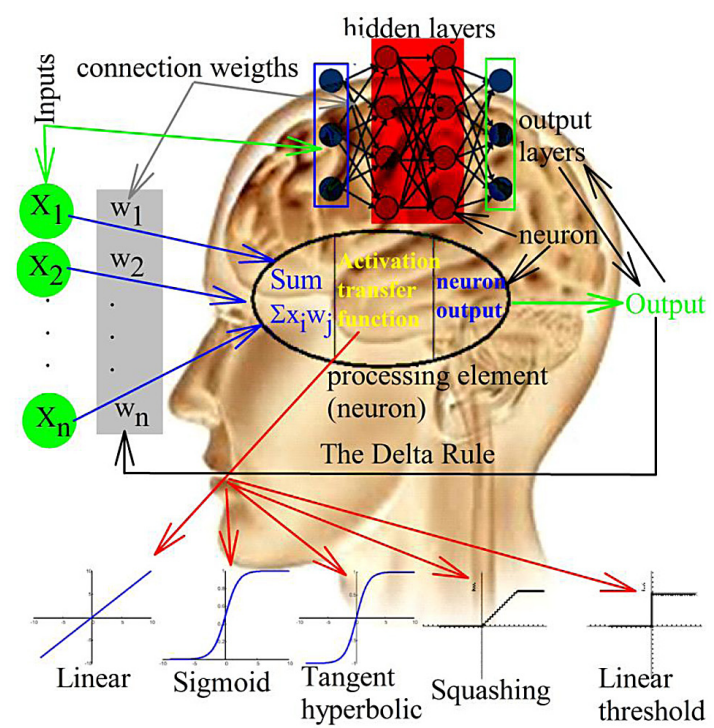

Fig. 2. Substituting the human brain with ANN computational model scheme and learning procedure

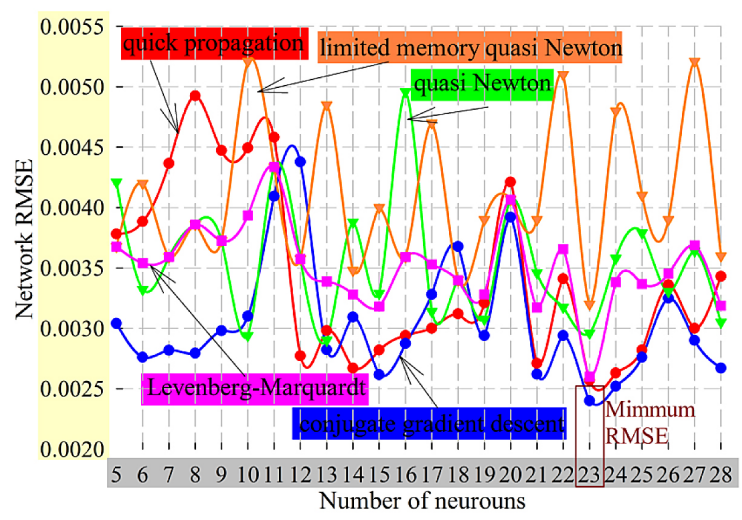

Fig. 3. Variation of RMSE for applied algorithms vs. number of neurons (the number of neurons 23 correspond to 7-7-5-5-3-3-1 showed the minimum RMSE)
Table 3. Range of used database in this paper

\begin{tabular}{|c|c|c|c|c|c|}
\hline Input & Min & Max & Input & Min & Max \\
\hline $\begin{array}{c}\text { Soil } \\
\text { type }\end{array}$ & $1(\mathrm{CL})$ & $5(\mathrm{SW}-\mathrm{SM})$ & $\sigma_{\mathrm{v}}$ & 13.55 & 451.34 \\
\hline$r_{d}$ & 0.566 & 0.983 & $\sigma_{v}^{\prime}$ & 4.53 & 331.53 \\
\hline $\begin{array}{c}\text { SPT-N } \\
\text { value }\end{array}$ & 5 & 50 & $\mathrm{~V}_{\mathrm{s}}$ & 217.49 & 358.262 \\
\hline \multicolumn{7}{|r|}{} & Depth & 0 & 80 \\
\hline
\end{tabular}

then logistic function. Then the operation was repeated and tested for the same structure using both logistic and hyperbolic tangent in different hidden layers. This operation process is repeated again for the same number of neurons but another topology and has been executed for all the number of neurons and then optimized ANN structure model was selected. The percentage of data for training, testing and validation with randomized selection were considered as 55\%, 25\% and 20\% respectively. The performance results of the optimized network for 3 runs and variation of MSE and standard deviations of both training and validation processes for 1000 epochs are given in Figure 5A, B and $\mathrm{C}$ respectively.

\section{RESULTS AND DISCUSSION}

The performance of the optimized ANN model can be controlled by mean absolute percentage error (MAPE), RMSE, variance account for (VAF), median absolute error (MEDAE), variance absolute relative error (VARE) statistical indices criteria as well as absolute error (AE) and absolute relative error (ARE). The formula- 


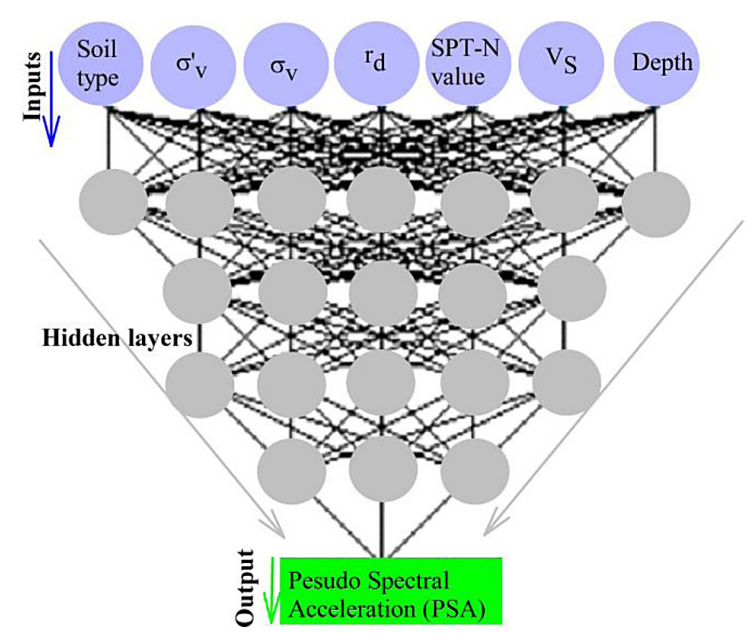

Fig. 4. Proposed ANN structure based model to predict the nonlinear site response spectrum in this study

tion of these indices can be found in statistical handbooks.

A model with higher coefficient of determination and VAF as well as lower RMSE, MAPE, VARE, MEDAE, AE and ARE will show better performance (Table 4 and Fig.6). The AE and ARE values define the deviation of the predicted output from the desired values. The AE is the difference between the actual and predicted values whereas the ARE is calculated by dividing the difference between actual and desired output values by the module of the desired output value. Both of AE and ARE correspond to model quality
Table 4. Results of statistical criteria for tested ANN algorithms

\begin{tabular}{|c|c|c|c|c|c|}
\hline Criteria & QP & CGD & QN & LMQN & L-M \\
\hline MAPE & 7.383 & $2.237(\mathrm{X})$ & 5.90 & 2.620 & 2.762 \\
\hline RMSE & 0.324 & $0.135(\mathrm{X})$ & 0.290 & 0.225 & 0.176 \\
\hline VARE & 16.30 & 6.54 & 11.83 & $5.28(\mathrm{X})$ & 6.64 \\
\hline MEDAE & 0.039 & $0.029(\mathrm{X})$ & 0.042 & 0.089 & 0.031 \\
\hline VAF & 95.88 & $98.28(\mathrm{X})$ & 96.73 & 96.97 & 97.80 \\
\hline R2 & 0.834 & $0.882(\mathrm{X})$ & 0.841 & 0.871 & 0.845 \\
\hline
\end{tabular}

and hence a smaller error indicates better performance in training.

Sensitivity analysis is a method to calculate the effectiveness of each input parameters on output. In the current paper two methods known as the Cosine Amplitude (Jong and Lee, 2004) and $\mathrm{PaD}$ (Gevrey et al., 2003) were used that both of them showed similar results but with different values (Table 5).

The site response analysis can be executed in one, two or three dimensional (1, 2 or $3 \mathrm{D})$. The $1 \mathrm{D}$ nonlinear site response analysis is mainly performed using time-domain employing nonlinear hysteretic soil models. However, this analysis requires a quantitative knowledge of actual nonlinear material behavior which can be obtained by sophisticated laboratory tests. Moreover, this approach needs deep understanding of analytical models and numerical methods. In comparison with 1D site response analysis, which needs
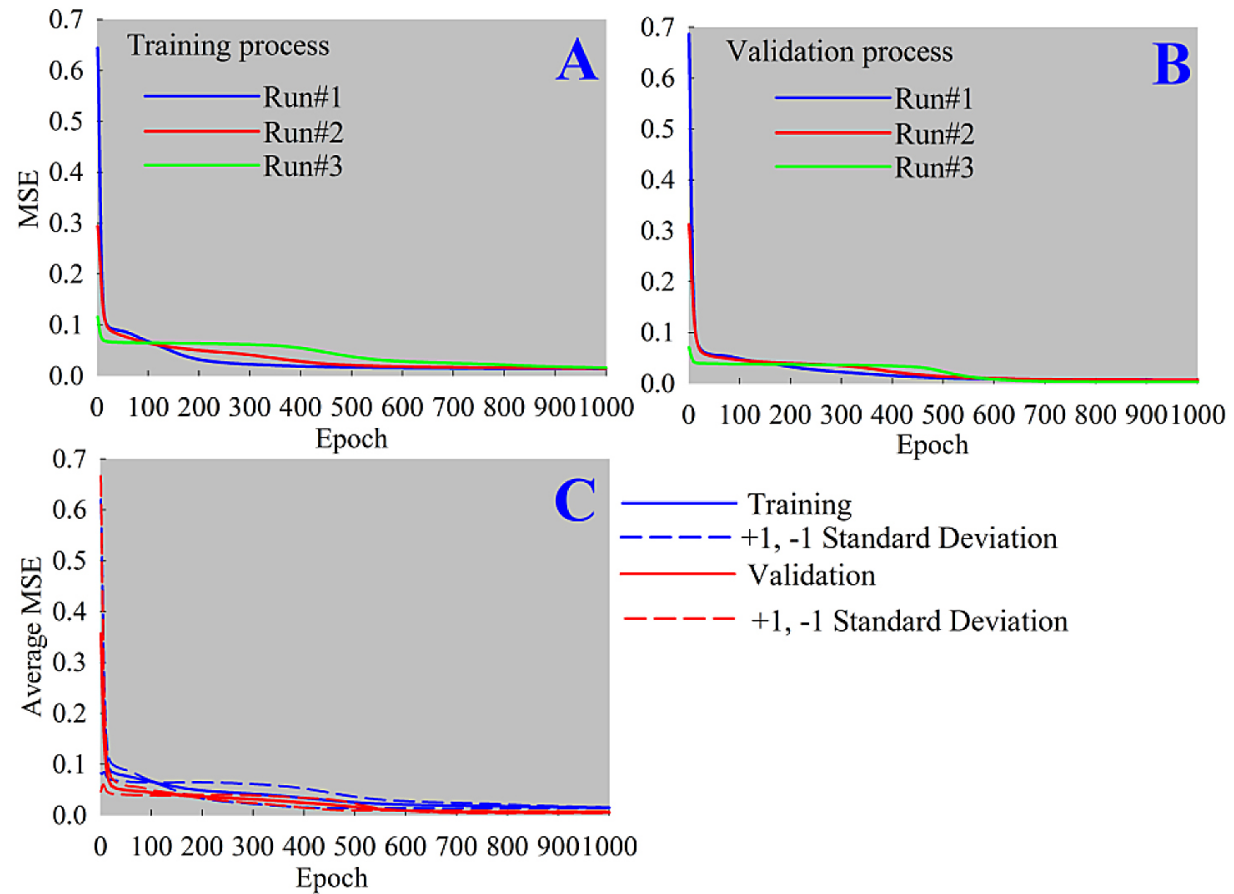

Epoch

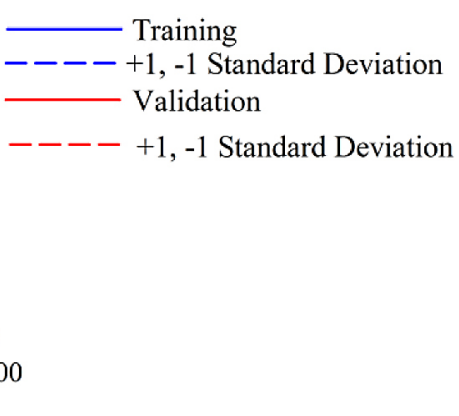

Fig. 5. Performance of introduced optimized ANN model in training and validation processes 

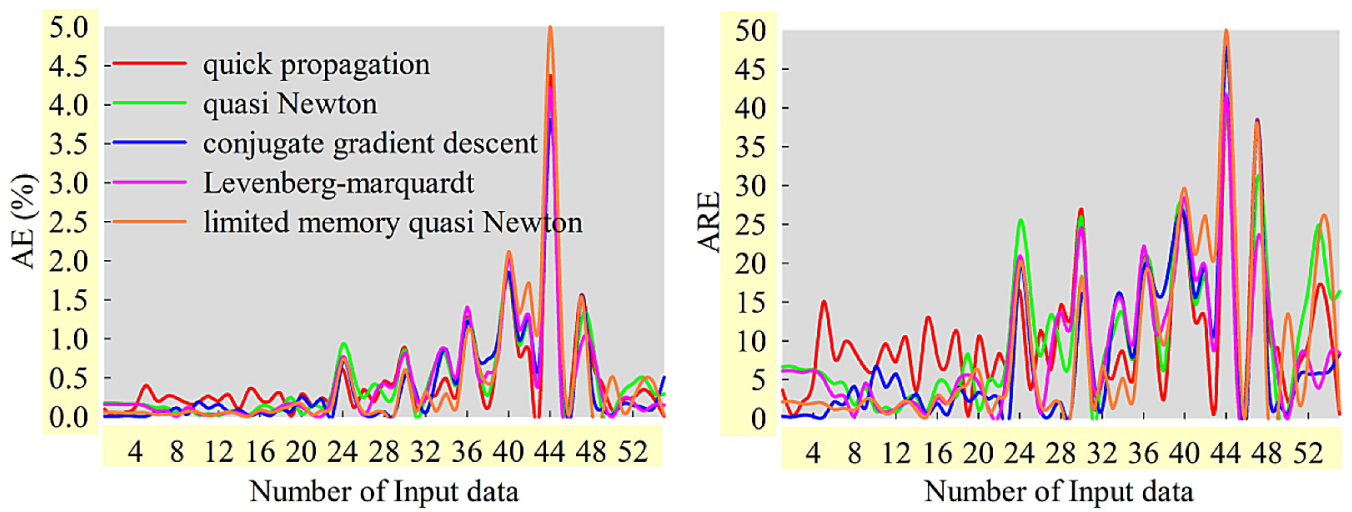

Fig. 6. Calculated AE and ARE (\%) of optimized ANN model for applied algorithms

Table 5. Influence of input parameters on output of optimized ANN model in this study

\begin{tabular}{|c|c|c|c|c|c|c|c|}
\hline \multirow{2}{*}{$\begin{array}{c}\text { Input } \\
\text { parameter }\end{array}$} & \multicolumn{7}{|c|}{ Importance (\%) } \\
\cline { 2 - 8 } & $\mathrm{V}_{\mathrm{s}}$ & Soil type & $\mathrm{r}_{\mathrm{d}}$ & $\sigma_{\mathrm{v}}^{\prime}$ & depth & SPT-N value & $\sigma_{\mathrm{v}}$ \\
\hline $\begin{array}{c}\text { Cosine } \\
\text { Amplitude }\end{array}$ & 34.81 & 33.12 & 31.12 & 28.10 & 27.86 & 26.77 & 12.87 \\
\hline $\mathrm{PaD}$ & 37.98 & 35.73 & 30.67 & 29.36 & 25.89 & 23.11 & 14.35 \\
\hline
\end{tabular}

geotechnical properties of the soil site (thickness, VS, unit weight), damping ratio, depth to bedrock, strong ground motion data, viscose damping formulation, soil model and backbone curves, the optimized ANN model in this study uses fewer types of input data. Furthermore, the used input parameters in the proposed ANN model can be obtained from routine in-situ or laboratory tests and or exact available formulation. Therefore, to evaluate the feasibility and applicability of ANN in site response prediction and also verify the obtained results from optimized ANN model, a comparison between ANN's outputs using different training algorithms regarding those obtained by numerical time domain, nonlinear analyses [25] were conducted (Figure 7A and B) and good com- patibilities were observed. In both Figure 7A and $\mathrm{B}$, the $\mathrm{Y}$-axis indicates a pseudo spectral acceleration (PSA) while in Figure 7A and B the $\mathrm{X}$-axis refers to the number of total dataset presented to ANN model and the period, respectively.

The PSA response spectrum provides a convenient and practical way to summarize the frequency content of a given acceleration, velocity or displacement time history. It provides a practical way to apply the knowledge of structural dynamics to design structures and the development of lateral force requirements in building codes. The PSA also provides a physically meaningful quantity, which is useful in understanding the nature of an earthquake and its influence on the design.
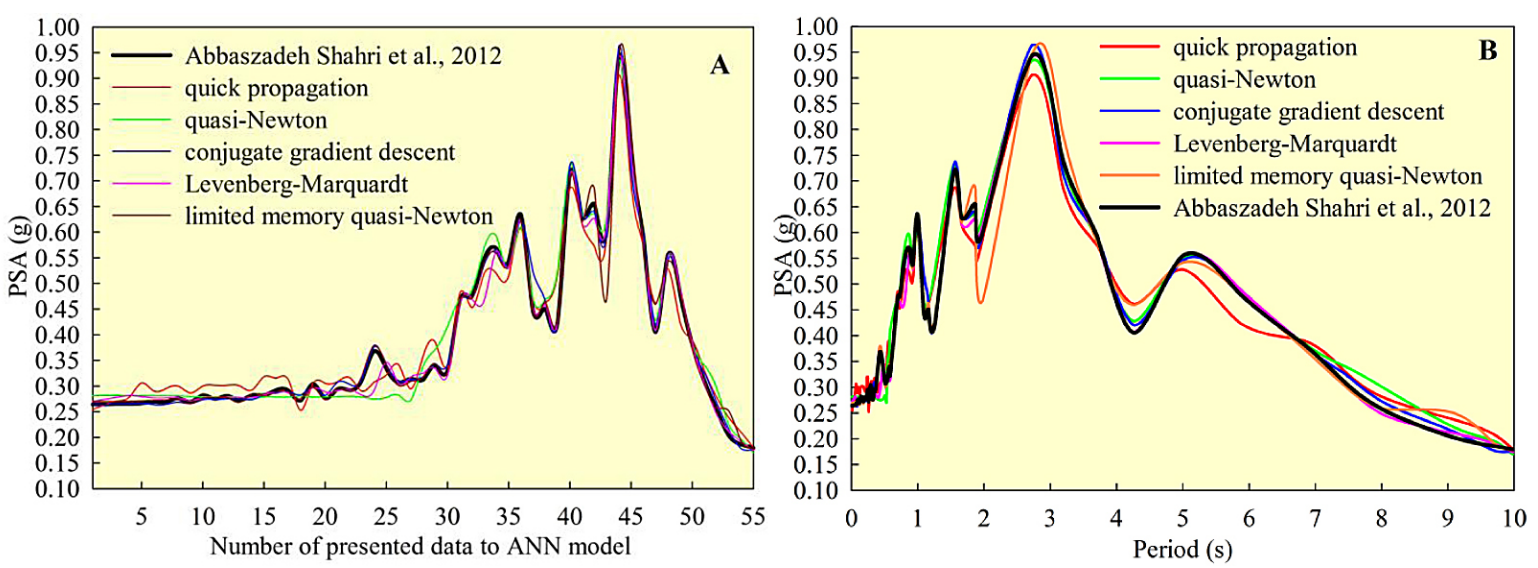

Fig. 7. Comparison of obtained results from tested ANN algorithms with numerical analysis result in the study area based on $(\mathrm{A})$ and $(\mathrm{B})$ period 


\section{CONCLUSIONS}

Estimating the soil site response spectrum, which is only applicable for characterized strong ground motion is costly and time consuming. In this study an alternative optimized and developed model using the ANN approach and application of different training algorithms was introduced to predict the $1 \mathrm{D}$ nonlinear seismic site response spectra. The proposed model was tested for a specified area in the west of Iran and its performance and quality evaluated by various criteria as well as comparison with numerical analyses. Moreover, the two applied different sensitivity analysis methods revealed similar results for the most and least effective factors on site response. Utilizing and using fewer input data, which can be obtained from routine in-situ or laboratory tests, as well as available exact formulation is the main advantage of presented ANN model respect to numerical analyses methods. In the input parameters, the soil types were coded in developed algorithm, what had not been done before.

The results highlighted a simpler, more effective and economical model, in comparison to the available complicated earthquake geotechnical procedures, which may require special software and data.

With the presented model in this study, there is a suitable and economic potential condition to reanalyze the used site response spectra of most early constructed dams in Iran, which suffer from the lack of information.

\section{REFERENCES}

1. Rubinstein JL. Nonlinear site response in medium magnitude earthquakes near Park field, California, Bull Seism Soc Am., 101 (1), 2011, 275-286.

2. Bonilla LF., Steidl JH., Gariel JC., Archuleta RJ. Borehole response studies at the Garner Valley Downhole Array, Southern California, Bull seism soc Am, 92, 2002, 3165-3179.

3. Abbaszadeh Shahri A., Behzadafshar k., Rajablou R., Verification of a new method for evaluation of liquefaction potential analysis. Arab J Geosci., 6 (3), 2013, 881-892.

4. Kottke AR. A comparison of seismic site response methods. Ph.D Dissertation, The University of Texas at Austin, Tx, USA, 2010.

5. Rodriguez-Marek A., Bray JD., Abrahamson NA. A geotechnical seismic site response evaluation procedure. In Proceeding of 12 WCEE, Auckland, New Zealand, 2000.
6. Vucetic M., Dobry R. Effect of soil plasticity on cyclic response. J Geotech Eng ASCE, 117 (1), 1991, 89-107.

7. Kramer SL. Geotechnical earthquake engineering. In prentice -Hall international series in civil engineering and engineering mechanics, Prentice-Hall, New Jersey, 1996.

8. Borcherdt RD. Effects of local geology on ground motion near San-Francisco-Bay. Bull Seism Soc Am., 60 (1), 1970, 29-61.

9. Seed HB., Ugas C., Lysmer J. Site-dependent spectra for earthquake-resistant design. Bull Seism Soc Am., 66 (1), 1976, 221-43.

10. Bard PY. Effects of surface geology on ground motion: recent results and remaining issues. In: Duma $\mathrm{G}$, editor. Proceeding of the 10th European conference on earthquake engineering. Rotterdam: AA Balkema, 1995, 305-323.

11. Tsang HH., Chandler AM. Site-specific probabilistic seismic-hazard assessment: direct amplitudebased approach. Bull Seism Soc Am., 96 (2), 2006, 392-403.

12. Chandler AM., Lam NTK., Sheikh MN. Response spectrum predictions for potential near-field and farfield earthquakes affecting Hong Kong: soil sites. Soil Dyn Earthquake Eng., 22, 2006, 419-440.

13. Tsompanakis Y., Lagaros ND., Psarropoulos PN., Georgopoulos EC. Simulating the seismic response of embankments via artificial neural networks. Advances in Engineering Software, 40, 2009, 640-651.

14. Kallassy A. A new neural network for response estimation. Comput Struct., 81 (26-27), 2003, 2417-2429.

15. Goh ATC. Nonlinear modeling in geotechnical engineering using neural networks. Australian Civil Engineering Transactions, CE36 (4), 1994, 293-297.

16. Hurtado JE., Londono JM., Meza MA. On the applicability of neural networks for soil dynamic amplification analysis. Soil Dyn Earthquake Eng., 21, 2001, 579-591.

17. Paolucci R., Colli P., Giacinto G. Assessment of seismic site effects in 2-D alluvial valleys using neural networks. Earthquake Spectra, 16 (3), 2002, 661-680.

18. Kerh T., Chu D. Neural networks approach and microtremor measurements in estimating peak ground acceleration due to strong motion. Adv Eng Softw., 33 (11-12), 2002, 733-742.

19. Kerh T., Ting SB. Neural network estimation of ground peak acceleration at stations along Taiwan high-speed rail system. Eng Appl Artif Intel., 18, 2005, 857-866.

20. Lin CCJ., Ghaboussi J. Generating multiple spectrum compatible accelerograms using stochastic 
neural networks. Earthquake Eng Struct Dyn., 30 (7), 2001, 1021-1042.

21. Seung CL., Sang WH. Neural-network-based models for generating artificial earthquakes and response spectra. Comput Struct., 80 (20-21), 2002, 1627-1638.

22. Lee SC., Han SW. Neural-network-based models for generating artificial earthquakes and response spectra. Computers \& Structures, 80 (20-21), 2002, 1627-1638.

23. Ghaboussi J., Lin CCJ. New method of generating spectrum compatible accelerograms using neural networks. Earthquake Engineering and Structural Dynamics, 27 (4), 1998, 377-396.

24. Kuzniar K., Waszczyszyn Z. Neural simulation of dynamic response of prefabricated buildings subjected to paraseismic excitations. Comput Struct., 81 (24-25), 2003, 2353-2360.

25. Abbaszadeh Shahri A., Esfandiyari B., Rajablou R. A proposed geotechnical-based method for evaluation of liquefaction potential analysis subjected to earthquake provocations (case study: Korzan earth dam, Hamedan province, Iran). Arab J Geosci., 5, 2012, 555-564.

26. Jackson JA., McKenzie D. Active tectonics of the Alpine-Himalayan Belt between western Turkey and Pakistan. Geophys. J. R. Astr. Soc., 77, 1984, 185-264.

27. Ni J., Barazangi M. Seismotectonics of the Zagros continental collision zone and a comparison with the Himalayas. J geophys Res, 91, 1986, 8205-8218

28. Wang RY., Strong D. What data quality means to data consumers. Journal of Management Information Sysytem, 12 (4), 1996, 5-34.

29. De Martin F. Influence of the nonlinear behavior of soft soils on strong ground motions. Ph.D dissertation, Engineering Sciences. Ecole Centrale, Paris, France, 2010.

30. Johnson P.A., Bodin P., Gomberg J.,Pearce F., Lawrence Z., Menq FR. Inducing in situ, nonlinear soil response applying an active source. Journal of Geophysical Research, 114, 2009, B05304.

31. Fausett L. Fundamentals of neural networks: architectures, and applications. Englewood Cliffs, NJ: Prentice-Hall, 1994.

32. Abbaszadeh Shahri A. An optimized artificial neural network structure to predict clay sensitivity in a high landslide prone area using piezocone penetration yest (CPTu) data: A case study in southwest of Sweden. Geotech Geol Eng., 2016, DOI 10.1007/ s10706-016-9976-y.

33. Jong YH., Lee CI. Influence of geological conditions on the powder factor for tunnel blasting. Int $\mathrm{J}$ Rock Mech Min Sci., 41 (1), 2004, 533-538

34. Gevrey M., Dimopoulos I., Lek S. Review and comparison of methods to study the contribution of variables in artificial neural network models. Ecological Modeling, 160 (3), 2003, 249-264. 